\title{
Nontrivial topology of cubic alkali bismuthides
}

\author{
I. P. Rusinov, ${ }^{1,2}$ I. Yu. Sklyadneva, ${ }^{3,4,5}$ R. Heid, ${ }^{4}$ K.-P. Bohnen, ${ }^{4}$ E. K. Petrov, ${ }^{1,2}$ Yu. M. Koroteev, ${ }^{5}$ \\ P. M. Echenique, ${ }^{3,6,7}$ and E. V. Chulkov ${ }^{2,3,6,7}$ \\ ${ }^{1}$ Tomsk State University, 634050 Tomsk, Russian Federation \\ ${ }^{2}$ St. Petersburg State University, 199034 St. Petersburg, Russian Federation \\ ${ }^{3}$ Donostia International Physics Center (DIPC), 20018 San Sebastián/Donostia, Basque Country, Spain \\ ${ }^{4}$ Karlsruher Institut für Technologie, Institut für Festkörperphysik, D-76021 Karlsruhe, Germany \\ ${ }^{5}$ Institute of Strength Physics and Materials Science, pr. Academicheskii 2/1, 634021 Tomsk, Russian Federation \\ ${ }^{6}$ Departamento de Física de Materiales, Facultad de Ciencias Químicas, UPV/EHU, Apartado 1072, \\ 20080 San Sebastián/Donostia, Basque Country, Spain \\ ${ }^{7}$ Centro de Física de Materiales CFM - Materials Physics Center MPC, Centro Mixto CSIC-UPV/EHU, 20018 San Sebastián/Donostia, Spain
}

(Received 27 January 2017; revised manuscript received 19 April 2017; published 28 June 2017)

\begin{abstract}
We report an ab initio study of the effect of pressure on vibrational and electronic properties of $\mathrm{K}_{3} \mathrm{Bi}$ and $\mathrm{Rb}_{3} \mathrm{Bi}$ in the cubic $F m \overline{3} m$ structure. It is shown that the high-temperature cubic phase of $\mathrm{K}_{3} \mathrm{Bi}$ and $\mathrm{Rb}_{3} \mathrm{Bi}$ is dynamically unstable at $T=0$ but can be stabilized by pressure. The electronic spectra of alkali bismuthides are found to possess the bulk band touching at the Brillouin zone center and an inverted spin-orbit bulk band structure. Upon hydrostatic compression the compounds transform from the topologically nontrivial semimetal $\left(\mathrm{K}_{3} \mathrm{Bi}\right) /$ metal $\left(\mathrm{Rb}_{3} \mathrm{Bi}\right)$ into a trivial semiconductor (metal) with a conical Dirac-type dispersion of electronic bands at the point of the topological transition. In $\mathrm{K}_{3} \mathrm{Bi}$ the dynamical stabilization occurs before the system undergoes the topological phase transition.
\end{abstract}

DOI: 10.1103/PhysRevB.95.224305

\section{INTRODUCTION}

The Bi-based binary compounds, $A_{3} \mathrm{Bi}(A=\mathrm{Na}, \mathrm{K}, \mathrm{Rb})$, have recently attracted much attention due to their unusual electronic properties. Using both first-principles calculations and effective model analysis [1], as well as angle-resolved photoemission spectroscopy (ARPES) [2,3], it was shown that in the low-temperature crystal structure (a hexagonal phase) these semimetals possess 3D Dirac states protected by crystal symmetry [4] with an inverted spin-orbit bulk band structure and nontrivial Fermi arcs on the surfaces $[1,5]$. Moreover, the compounds can be driven into various topologically distinct phases, such as topological insulator, topological metal (with nontrivial Fermi surfaces), or Weyl semimetal by breaking of symmetry [6].

On the other hand, it is well known that $\mathrm{K}_{3} \mathrm{Bi}$ and $\mathrm{Rb}_{3} \mathrm{Bi}$ undergo a reversible transition from a hexagonal low-temperature phase to a cubic one, $F m \overline{3} m$, at $280^{\circ} \mathrm{C}$ and $230^{\circ} \mathrm{C}$, respectively $[7,8] . \mathrm{Na}_{3} \mathrm{Bi}$, which crystallizes in a hexagonal structure regardless of temperature, becomes cubic under hydrostatic compression [9,10]. As shown in Ref. [11] and in the present work, in the cubic structure these compounds are also semimetals with an inverted band order between the conduction and valence bands at the center of the Brillouin zone (BZ). Such materials can be driven to topologically different phases by pressure-induced modifications of the band structure [12]. The prediction of new topological phases by using $a b$ initio computational tools has become an effective way to search materials with new properties. The unique electronic structure of the compounds also opens up opportunities to study topological phase transitions. The conical dispersion in such Dirac semimetals is realized at the critical point of a topological phase transition and is achieved by tuning of a system parameter (pressure), which makes the band structure easy to engineer suitably $[13,14]$.
Inspired by recent theoretical studies of $\mathrm{Na}_{3} \mathrm{Bi}$ and using first-principles band-structure calculations, we show that upon hydrostatic compression cubic $\mathrm{K}_{3} \mathrm{Bi}$ and $\mathrm{Rb}_{3} \mathrm{Bi}$ undergo a transition from a topological semimetal $\left(\mathrm{K}_{3} \mathrm{Bi}\right)$-metal $\left(\mathrm{Rb}_{3} \mathrm{Bi}\right)$ to a conventional semiconductor $\left(\mathrm{K}_{3} \mathrm{Bi}\right)$-metal $\left(\mathrm{Rb}_{3} \mathrm{Bi}\right)$. To examine the dynamical stability of $\mathrm{K}_{3} \mathrm{Bi}$ and $\mathrm{Rb}_{3} \mathrm{Bi}$ in the cubic phase and the effect of pressure on the lattice dynamics, we have calculated the phonon dispersion with and without hydrostatic compression.

\section{CAlCulation Details}

The electronic structure calculations were performed in the mixed-basis (MB) pseudopotential approach [15-17] with the exchange and correlation energy functional evaluated within the generalized gradient approximation [18]. We used semilocal norm-conserving pseudopotentials [19], with $p$ semicore states treated as valence states for alkali-metal atoms ( $3 p$ for $\mathrm{K}$ and $4 p$ for $\mathrm{Rb}$ ). The $\mathrm{MB}$ scheme employs a combination of local functions and plane waves. To expand valence states, we used a plane wave basis set with a kinetic energy cutoff of $22 \mathrm{Ry}(\sim 300 \mathrm{eV})$ augmented by local $p$ - and $d$-type functions for alkali-metal atoms and $s$ - and $p$-type local functions for $\mathrm{Bi}$ at each atomic site. The Fourier expansion of the crystal potential and charge density was found to be safely truncated at $54 \mathrm{Ry}$. Integrations over the Brillouin zone (BZ) were performed by sampling an $8 \times 8 \times 8$ mesh corresponding to $512 k$ points in the $\mathrm{BZ}$ and using a Gaussian broadening with a smearing parameter of $0.01 \mathrm{eV}$.

To obtain phonon dispersions, the density-functional perturbation theory was used [20]. Its implementation in the MB scheme is described in Ref. [17]. We performed both scalar relativistic and including-spin-orbit-effect calculations. Spin-orbit coupling (SOC) was incorporated into the 
pseudopotential scheme via the Kleinman's formulation [21] and was treated fully self-consistently [22]. The dynamical matrices were computed on a $(4 \times 4 \times 4)$ grid of wave vectors, and then the phonon frequencies along high-symmetry directions of the BZ were obtained with the standard Fourier interpolation scheme [23].

\section{A. Wilson loop method}

To demonstrate the topological character of the compounds, we applied the Wilson loop (also known as Wannier charge center) method, proposed in Refs. [24,25]. The Wilson loop $(\hat{\mathcal{W}})$ is defined as

$$
\hat{\mathcal{W}}_{\mu}=\mathcal{P} \exp \left[-i \int_{-\pi}^{\pi} d \mathbf{k} \hat{\mathcal{A}}_{\mu}(\mathbf{k})\right]
$$

where $\mathcal{P}$ is the path-ordering operator. $\hat{\mathcal{A}}(\mathbf{k})$ is the non-Abelian Berry connection,

$$
\hat{\mathcal{A}}_{\mu}^{m n}(\mathbf{k})=-i\left\langle u_{m \mathbf{k}}\left|\partial_{k_{\mu}}\right| u_{n \mathbf{k}}\right\rangle,
$$

where $m, n$ are band indexes, $\mu$ is a space index, and $u_{n \mathbf{k}}$ are the Bloch eigenstates of the Hamiltonian. Practically, a tight-binding Wilson loop and discretized expression [26] are used,

$$
\hat{\mathcal{W}}_{\mu}^{m n}=\left\langle u_{m}\left(\mathbf{k}_{0}\right)\left|\prod_{\mathbf{k}=\mathbf{k}_{0}}^{\mathbf{k}_{0}+\mathbf{G}_{\mu}} P^{o c c}(\mathbf{k})\right| u_{n}\left(\mathbf{k}_{0}+\mathbf{G}_{\mu}\right)\right\rangle,
$$

where $P^{o c c}(\mathbf{k})=\sum_{i=1}^{o c c}\left|u_{i}(\mathbf{k})\right\rangle\left\langle u_{i}(\mathbf{k})\right|, u_{i}(\mathbf{k})=u_{i}\left(\mathbf{k}+\mathbf{G}_{\mu}\right)$ for each $\mu$, and $\mathbf{G}_{\mu}$ are basis vectors in the reciprocal space. The product is found on a finite mesh of closely adjacent $\mathbf{k}$ points which form a closed loop line. It should be noted that this line is perpendicular to the direction of $\mathbf{k}$ points where Wilson loops are calculated. The eigenspectrum of $\hat{\mathcal{W}}_{\mu}^{m n}$ defines Wannier charge centers and makes it possible to graphically investigate the topology of compounds.

\section{RESULTS AND DISCUSSION}

\section{A. Structural parameters}

The high-temperature phase of $\mathrm{K}_{3} \mathrm{Bi}$ and $\mathrm{Rb}_{3} \mathrm{Bi}, F m \overline{3} m$, is composed of four face-centered sublattices mutually shifted by $\left(\frac{1}{4}, \frac{1}{4}, \frac{1}{4}\right)$ along the body diagonal with two of them being symmetry equivalent. Atomic positions are shown in Table I. Also shown are the optimized lattice parameter obtained by total energy minimization together with available experimental data. In $\mathrm{K}_{3} \mathrm{Bi}$, the theoretical lattice parameter is very close to the experimental value, the deviation is $\sim 0.15 \%$. For $\mathrm{Rb}_{3} \mathrm{Bi}$ a

TABLE I. Positions of atoms (O, octahedral hole; T, tetrahedral hole) and lattice parameters (in $\AA$ ). The theoretical lattice constants, $a^{\text {th }}$, were obtained with the account of SOC. The experimental parameters, $a^{\text {expt }}$, are taken from Refs. [7,27].

\begin{tabular}{lcccc}
\hline \hline $\mathrm{K}(\mathrm{Rb})_{3} \mathrm{Bi}$ & Position & & $a^{\text {th }}$ & $a^{\text {expt }}$ \\
\hline $\mathrm{K}(\mathrm{Rb})^{\mathrm{O}}$ & $(1 / 2,1 / 2,1 / 2)$ & $\mathrm{K}_{3} \mathrm{Bi}$ & 8.818 & 8.805 \\
$\mathrm{~K}(\mathrm{Rb})^{\mathrm{T}}$ & $\pm(1 / 4,1 / 4,1 / 4)$ & $\mathrm{Rb}_{3} \mathrm{Bi}$ & 9.218 & 8.98 \\
$\mathrm{Bi}$ & $(0,0,0)$ & & & \\
\hline \hline
\end{tabular}

larger underbinding of $\sim 2.6 \%$ is obtained. Such a deviation is rather typical for generalized gradient approximation-PerdewBurke-Ernzerhof calculations.

\section{B. Dynamical stability}

The phonon spectra of $\mathrm{K}_{3} \mathrm{Bi}$ and $\mathrm{Rb}_{3} \mathrm{Bi}$ obtained at the equilibrium volume $\left(V_{0}\right)$ show that the cubic phase is dynamically unstable at zero temperature. The phonon dispersions are given in Figs. 1(c) and 1(d). The right panels show the density of phonon states. Since the unit cell contains four atoms, there are 12 vibrational modes. The harmonic calculation yields imaginary phonon frequencies at the BZ boundary for two transverse acoustic (TA) modes around the $X$ point and for one TA mode near the $\mathrm{K}$ and $\mathrm{U}$ symmetry points. The modes are shown as negative ones in the figure. The corresponding atomic displacements are given schematically in Figs. 1(e) and 1(f). The unstable modes are dominated by displacements of those $\mathrm{K}(\mathrm{Rb})$ atoms which occupy octahedral sites $\left[\mathrm{K}(\mathrm{Rb})^{\mathrm{O}}\right.$; see Table I].

To explore the effect of pressure, we have calculated the phonon spectra of $\mathrm{K}_{3} \mathrm{Bi}$ and $\mathrm{Rb}_{3} \mathrm{~B}$ at different volumes. It is found that upon volume decrease, all phonon modes experience an upward shift and at a volume of $V \sim 0.92 V_{0}(P \sim$ $1 \mathrm{GPa})$ in $\mathrm{K}_{3} \mathrm{Bi}$ and at $V \sim 0.82 V_{0}[P \sim 2.5 \mathrm{GPa}(\sim 1 \mathrm{GPa})$ with respect to $\left.V_{0}\left(V_{\exp }\right)\right]$ in $\mathrm{Rb}_{3} \mathrm{Bi}$ the TA modes in the phonon spectrum become stable. It means that at low temperatures the cubic $F m \overline{3} m$ phase of $\mathrm{K}_{3} \mathrm{Bi}$ and $\mathrm{Rb}_{3} \mathrm{Bi}$ is stabilized by pressure. Another alkali bismuthide, $\mathrm{Na}_{3} \mathrm{Bi}$, also becomes cubic under hydrostatic compression $[9,10]$ (at $0.7-1.0 \mathrm{GPa}$ ) although the compound crystallizes in a hexagonal structure regardless of temperature. Unlike $\mathrm{K}_{3} \mathrm{Bi}$ and $\mathrm{Rb}_{3} \mathrm{Bi}$, the cubic structure of $\mathrm{Na}_{3} \mathrm{Bi}$ is still dynamically stable at ambient pressure, as shown by lattice dynamics calculations [10].

To show the structural transition under pressure, we have also calculated the enthalpy difference between the cubic and the hexagonal phases. The calculations were carried out within the projected augmented-wave approach realized in the VASP code [28]. Lattice parameters and atomic positions were optimized for each pressure using the L-BFGS algorithm to solve unconstrained minimization problems [29]. The data for three possible hexagonal phases are presented in Fig. 2. Like $\mathrm{Na}_{3} \mathrm{Bi}$, both compounds were first found to crystallize in the $\mathrm{P6}_{3} / m m c$ structure ( $\mathrm{Na}_{3} \mathrm{As}$-type) [30]. For $\mathrm{K}_{3} \mathrm{Bi}$, two other hexagonal phases were then reported: $P \overline{3} c 1$ (anti-LaF $\mathrm{La}_{3}$-type, Ref. [31] and $P 6_{3} \mathrm{~cm}$ ( $\mathrm{Cu}_{3} \mathrm{P}$-type; Ref. [32]). As compared to the $P 6_{3} / \mathrm{mmc}$ phase, the unit cell of $P \overline{3} \mathrm{c} 1$ and $P 6_{3} \mathrm{~cm}$ structures is increased from 8 atoms per unit cell to 24 as a result of a distorted $\mathrm{K}(\mathrm{Rb})-\mathrm{Bi}$ honeycomb lattice [33]. The calculated enthalpies show that (i) the $P \overline{3} c 1$ and $P 6_{3} \mathrm{~cm}$ hexagonal structures are thermodynamically preferable when compared to the earlier reported $P 6_{3} / m m c$ phase: $\Delta H(P=$ 0 ) for $P \overline{3} \mathrm{c} 1$ and $P 6_{3} \mathrm{~cm}$ is about $0.01 \mathrm{meV} /$ f.u. (in $\mathrm{K}_{3} \mathrm{Bi}$ ) and $0.04 \mathrm{meV} /$ f.u. (in $\mathrm{Rb}_{3} \mathrm{Bi}$ ) smaller as compared to that for the $\mathrm{P}_{3} \mathrm{~cm}$ structure; (ii) the structural transition to the cubic phase indeed occurs above $\sim 1 \mathrm{GPa}$ (with respect to the experimental volume). For $\mathrm{Na}_{3} \mathrm{Bi}$, the $P \overline{3} c 1$ phase was also experimentally confirmed by Raman measurements [34]. The observed number of IR active modes ruled out the $P 6_{3} / m m c$ symmetry. 

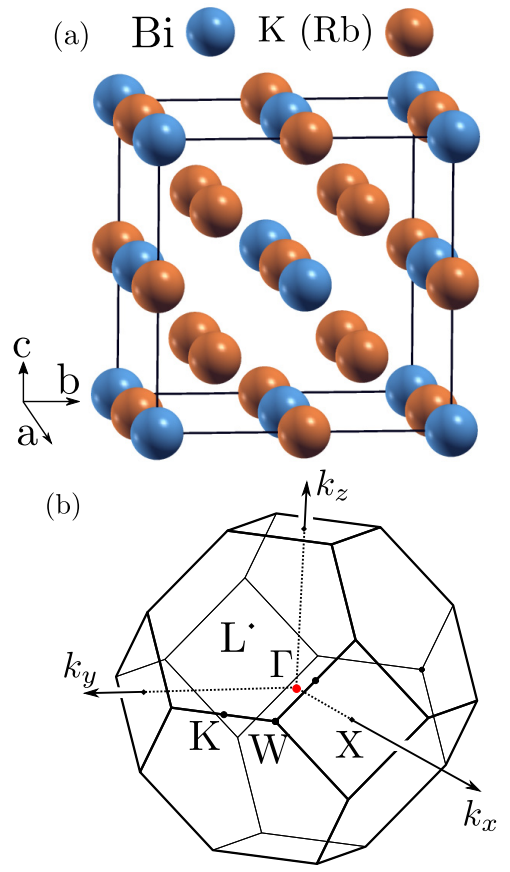
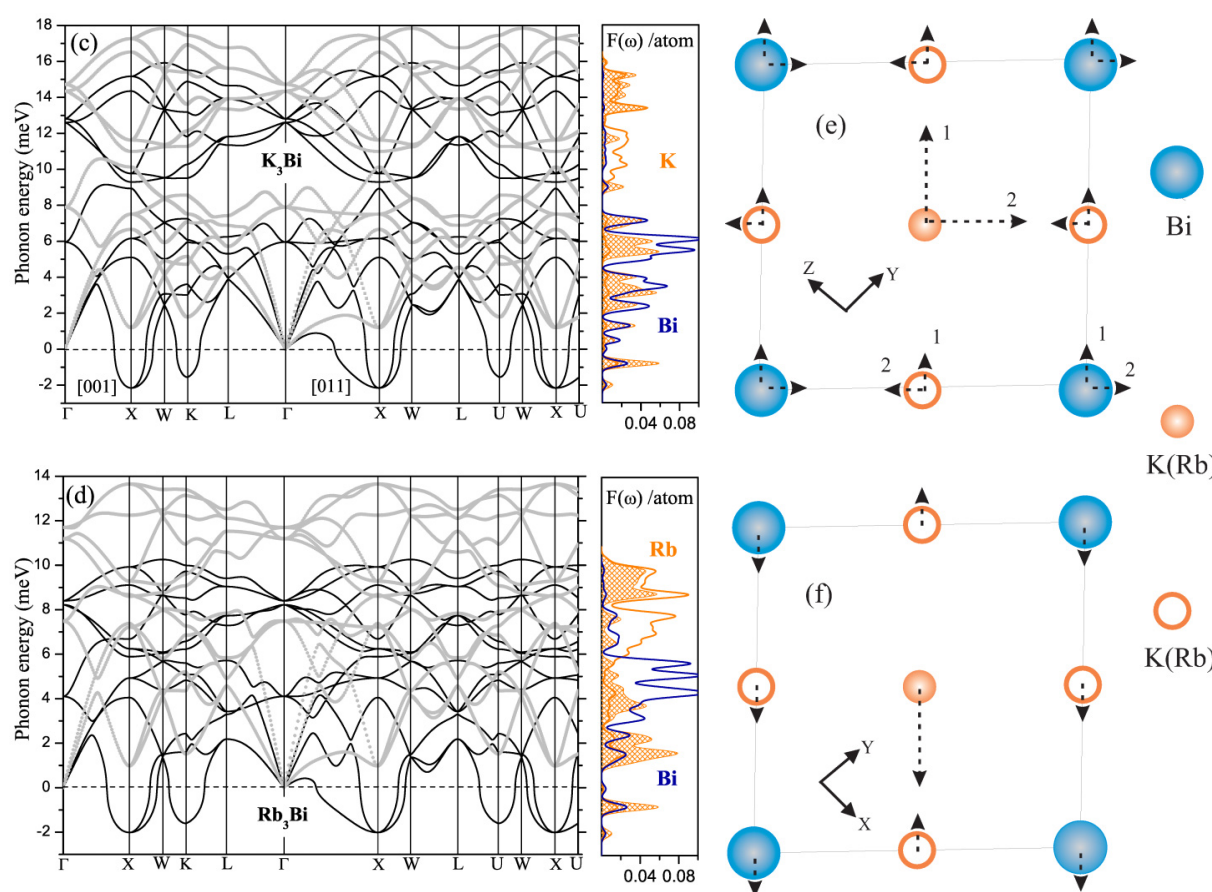

(

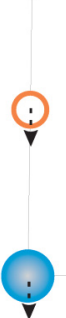

(f)

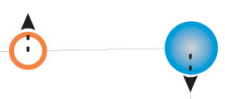

$\mathrm{K}(\mathrm{Rb})^{\circ}$

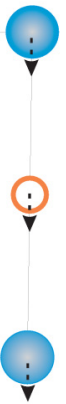

FIG. 1. (a) Atomic positions and (b) high-symmetry points in the bulk Brillouin zone. (c),(d) Phonon dispersions of $\mathrm{K}_{3} \mathrm{Bi}(\mathrm{c})$ and $\mathrm{Rb} \mathrm{b}_{3} \mathrm{Bi}$ (d) calculated with SOC are shown by black lines for the equilibrium theoretical volume $V_{0}$ and by gray lines for $V / V_{0} \approx 0.9\left(\mathrm{~K}_{3} \mathrm{Bi}\right)$ and $V / V_{0} \approx 0.8\left(\mathrm{Rb}_{3} \mathrm{Bi}\right)$. The right panels show the density of phonon states at $V_{0}$. The contributions coming from the motion of different atoms are given in corresponding colors. (e),(f) Atomic displacements for two TA modes at X (e) and one TA mode at K (d). Alkali-metal atoms are shown by solid (in the octahedral sites) and open (in the tetrahedral sites, they are out of the figure plane) circles.

\section{Electronic structure}

\section{Electronic structure at ambient pressure}

The calculated electronic spectra of $\mathrm{K}_{3} \mathrm{Bi}$ and $\mathrm{Rb}_{3} \mathrm{Bi}$ in the low-temperature $P \overline{3} c 1$ structure (a hexagonal phase) are shown in Figs. 3(a) and 3(d). The electronic bands were obtained with SOC included. The band dispersions are similar to those reported for the $P 6_{3} / m m c$ structure [6]. As follows from the figure, the hexagonal $\mathrm{K}_{3} \mathrm{Bi}$ and $\mathrm{Rb}_{3} \mathrm{Bi}$ are metallic with small electron pockets around the $\Gamma$ point, while $\mathrm{Na}_{3} \mathrm{Bi}$ is a semimetal $[1,6,33]$. Like in the case of $\mathrm{Na}_{3} \mathrm{Bi}$, the conduction and valence bands are inverted at the bulk BZ center where the $\mathrm{K} / \mathrm{Rb} s$ band is located at lower energy than the $\mathrm{Bi} p_{x}, p_{y}$ band. The band inversion at the $\Gamma$ point indicates a topologically nontrivial character of the electronic structure, which is also

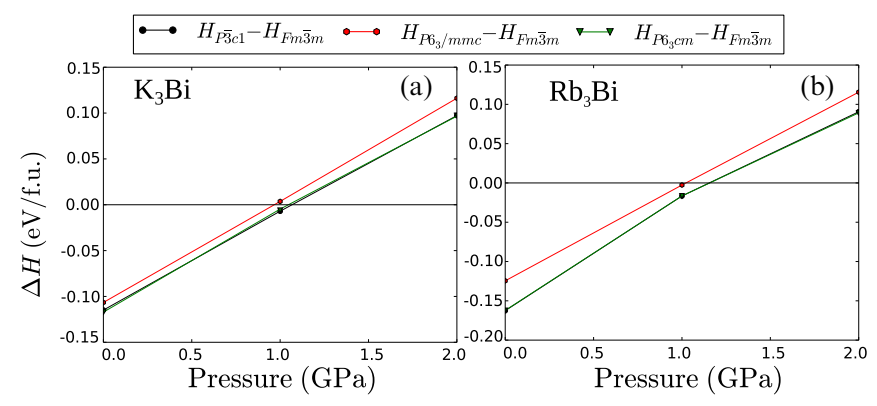

FIG. 2. Pressure-dependent enthalpy per formula unit (f.u.) of the hexagonal structures relative to that of the cubic one $(\Delta H)$ for $\mathrm{K}_{3} \mathrm{Bi}$ (a) and $\mathrm{Rb}_{3} \mathrm{Bi}(\mathrm{b})$. manifested in the existence of topological surface states. The states with a Dirac-type crossing at the $\bar{\Gamma}$ point are clearly seen on the (0001) surface [see Figs. 3(b) and 3(e)] inside the projected valence bands. However, another feature, a pair of Dirac nodes (band crossings) at the Fermi level on the rotational $\mathbf{k}_{z}$ axis (the $\Gamma$-A symmetry direction in momentum space), shows up only in $\mathrm{K}_{3} \mathrm{Bi}$. The crossing points are fourfold degenerate due to the time-reversal and inversion symmetries.

The bulk Dirac nodes on the rotational axis away from the $\Gamma$ point indicate that the Dirac states are symmetry-protected by the threefold rotation crystal symmetry [3] which remains in the $P \overline{3} c 1$ structure although the $\mathrm{K} / \mathrm{Bi}$ sheets are buckled due to the shifting of alkali-metal atoms $[1,6,33]$. The projected bulk Dirac nodes and the nontrivial surface states (Fermi arcs) can be visible on the $\mathrm{K}_{3} \mathrm{Bi}(01 \overline{1} 0)$ surface [see Fig. 3(c)], where they are separated in the momentum space. So $\mathrm{K}_{3} \mathrm{Bi}$ in the low-temperature hexagonal phase is a $3 \mathrm{D}$ topological Dirac metal with two Dirac cones. Unlike $\mathrm{K}_{3} \mathrm{Bi}$, the electronic spectrum of $\mathrm{Rb}_{3} \mathrm{Bi}$ shows only a band inversion in the $\mathrm{BZ}$ center. We could not find any indication of a Dirac-type band crossing on the rotational axis along the $\Gamma$-A line [Fig. 3(f)].

It should be noted that the electronic properties of alkali bismuthides in the cubic structure have been studied before within semirelativistic calculations [35]. Figure 4 shows the electronic spectra of $\mathrm{K}_{3} \mathrm{Bi}$ and $\mathrm{Rb}_{3} \mathrm{Bi}$ obtained with the inclusion of spin-orbit coupling (a) and (b) and also displays the influence of SOC on the electronic bands (c) and (d) in the vicinity of the BZ center. Without taking into account the spin-orbit coupling, the $\mathrm{K}_{3} \mathrm{Bi}$ compound is a trivial semimetal with a small gap (see Table II) at the $\Gamma$ point. The electronic 
(a)

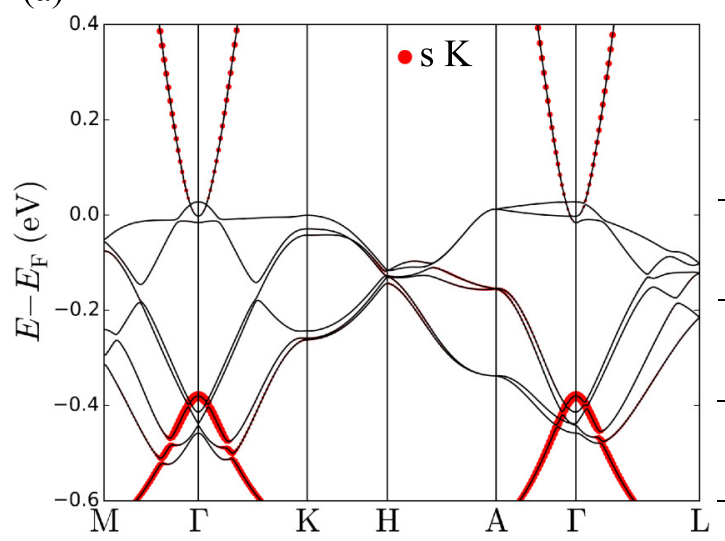

(d)

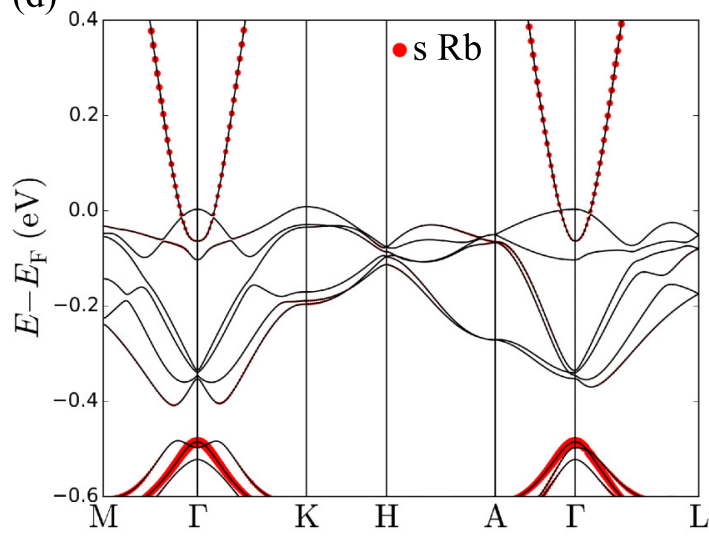

(b)

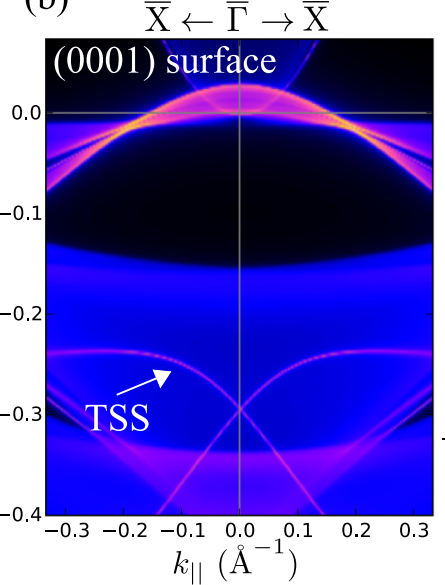

(e)

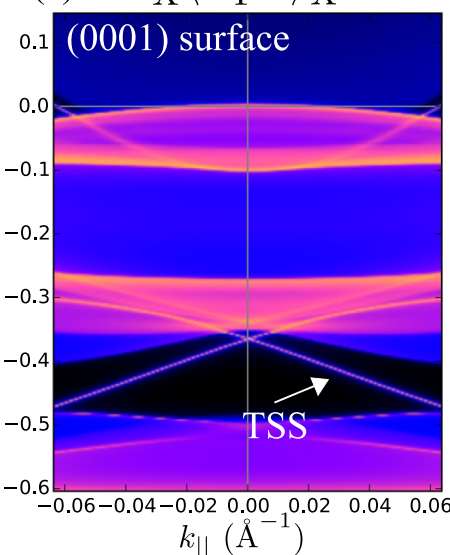

(c) $\overline{\mathrm{X}} \leftarrow \bar{\Gamma} \rightarrow \overline{\mathrm{Z}}$

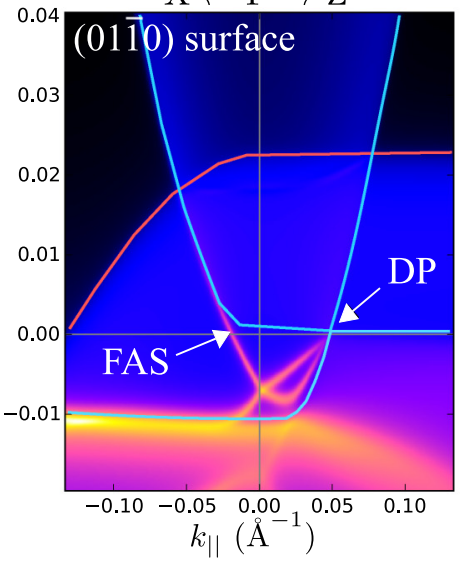

(f)

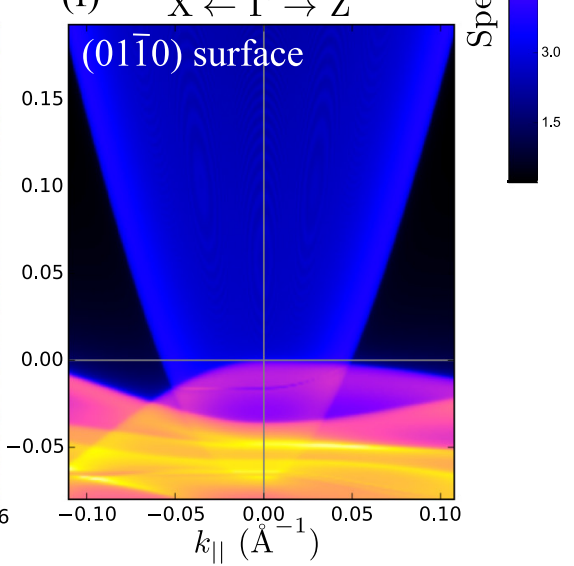

FIG. 3. Electronic bands of (a) $\mathrm{K}_{3} \mathrm{Bi}$ and (d) $\mathrm{Rb}_{3} \mathrm{Bi}$ in the hexagonal $(P \overline{3} c 1)$ structure. Red circles (lines) indicate $\mathrm{K}(\mathrm{Rb}) s$ states. (b),(c),(e),(f) Projected spectral functions calculated at the equilibrium volume for the (0001) and (0110) surfaces of $\mathrm{K}_{3} \mathrm{Bi}(\mathrm{b}),(\mathrm{c})$ and $\mathrm{Rb}{ }_{3} \mathrm{Bi}$ (e),(f). The projection is shown by a color scale reflecting the localization in the outermost surface layer (in units of states/eV). TSS means a topological surface state, DP means a Dirac point, and FAS is a Fermi arc state.

structure of $\mathrm{Rb}_{3} \mathrm{Bi}$ is characterized by a small electron pocket around $\Gamma$ and a hole pocket in the vicinity of the $X$ point. In both compounds the lowest conduction band at the $\Gamma$ point is composed of $s$-type electronic states and is located slightly above the three $p$-like bands, which are degenerate at the BZ center. Accounting of SOC modifies the dispersion of valence bands by splitting off the $p$ bands at $\Gamma$ into two degenerate bands, $\Gamma_{8}$, and a split-off state, $\Gamma_{7}$ so that the valence and conduction bands are now touching at the BZ center. SOC also affects the $s$-type electronic states, band $\Gamma_{6}$; they are pushed below the Bi $p$ bands, $\Gamma_{8}$. As a result the energy gap $\varepsilon^{S}\left(\Gamma_{6}\right)-\varepsilon^{P}\left(\Gamma_{8}\right)$ becomes negative and thereby the band order at the BZ center gets inverted, which indicates a topologically nontrivial phase. Since the theoretical and experimental lattice parameters differ slightly, Table II shows the gap values for both lattice constants. In addition, it is worth noting that no other band inversions are present for the rest of the highsymmetric points in the Brillouin zone. The band inversion in $\mathrm{K}_{3} \mathrm{Bi}$ and $\mathrm{Rb}_{3} \mathrm{Bi}$ is confirmed by using an improved approximation for the exchange energy, the hybrid exchangecorrelation functional HSE06. It is based on a screened Coulomb potential for the exchange interaction to screen the long-range part of the Hartree-Fock (HF) exchange [36,37]. The HF term with the correct asymptotic behavior makes it possible to improve the accuracy of the band-structure prediction [38].

$\mathrm{Na}_{3} \mathrm{Bi}$ in the strain-free cubic case is also a semimetal like $\mathrm{K}_{3} \mathrm{Bi}$ because its conduction and valence bands touch only at the $\Gamma$ point. However, even in the case without SOC the $\mathrm{Na}-3 s$ states are energetically lower, by about $0.4 \mathrm{eV}$, than the $\mathrm{Bi}-6 p$ states at the $\Gamma$ point [11] and, what is more important, the $s$ and $p$ states exhibit the opposite parities. When the SOC is taken into account, the inverted band ordering is further enhanced. The $\mathrm{Na}-3 s$ state $\left(\Gamma_{6}\right)$ is significantly reduced in energy, thereby increasing the energy difference with $\mathrm{Bi} p$ states up to $0.85 \mathrm{eV}$. The same characteristic feature was reported for a ternary alkali bismuthide, $\mathrm{KNa}_{2} \mathrm{Bi}$ [39]. Therefore, like $\mathrm{KNa}_{2} \mathrm{Bi}, \mathrm{Na}_{3} \mathrm{Bi}$ in its strain-free cubic case is a topologically nontrivial zero-gap semimetal at $P=0 \mathrm{GPa}$, whether or not the SOC is considered.

To demonstrate the topological character of the considered compounds, we also applied the Wilson loop (Wannier charge center) method, proposed in Refs. [24,25]. Wannier charge centers (WCCs) were obtained from the occupied states on the basis of the WANNIER90 code Hamiltonian [40]. The topological properties were investigated by checking two planes in the $k$ space: $k_{z}=0$ and $k_{z}=\pi$. The evolution of WCC 

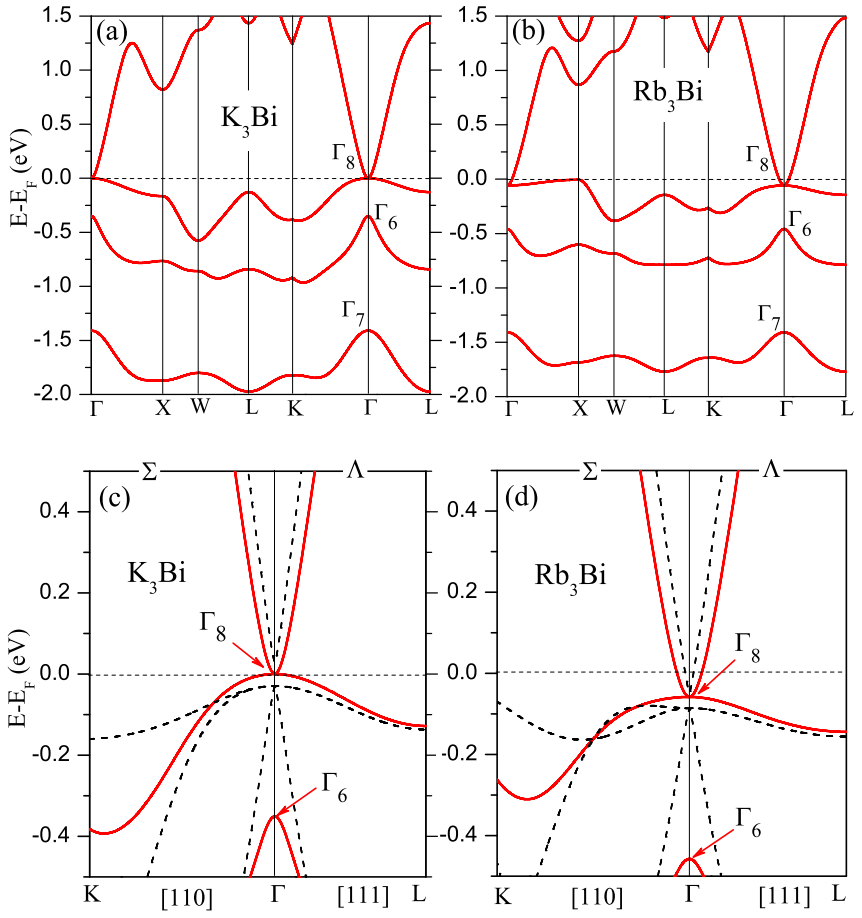

FIG. 4. Electronic band structures of (a) $\mathrm{K}_{3} \mathrm{Bi}$ and (b) $\mathrm{Rb}_{3} \mathrm{Bi}$ calculated with the account of SOC. (c),(d) Electronic bands of $\mathrm{K}_{3} \mathrm{Bi}$ and $\mathrm{Rb}_{3} \mathrm{Bi}$ obtained without (dashed lines) and with the inclusion of spin-orbit coupling (red solid lines) along the [110] $(\Sigma)$ and [111] $(\Lambda)$ symmetry directions.

$(\theta)$ for $k_{x} \in[0, \pi]$ at fixed $k_{z}=0$ (left) and $k_{z}=\pi$ (right) is shown in Fig. 5 for $\mathrm{K}_{3} \mathrm{Bi}$. For each $k_{x}$, overlap matrices have been obtained along string $k_{y} \in[-\pi, \pi]$. At $k_{x}=\pi n(n$, integer) all the presented curves are doubly degenerate due to time-reversal symmetry in the considered systems. Owing to the point symmetry, the results are similar for perpendicular planes when $k_{x}$ and $k_{y}$ are fixed and the evolution of WCC is traced along the $k_{y}$ and $k_{z}$ directions, respectively. From the figure it is clear that for the $k_{z}=0$ plane the WCC curve crosses the reference line once. However, WCC never crosses the reference line at $k_{z}=\pi$. It should be noted that the reference line parallel to the $k_{x}$ axis is purely arbitrary and can be moved to somewhere else, but the parity of crossing numbers between the evolution lines and the reference line will never change. This indicates the topological character of $\mathrm{K}_{3} \mathrm{Bi}$ and agrees with the band inversion at the $\Gamma$ point. The presented WCC curves also say that $Z_{2}$ is equal to $(1 ; 000)$ in the

TABLE II. Calculated energy gaps at the $\Gamma$ point, $\varepsilon^{S}\left(\Gamma_{6}\right)-$ $\varepsilon^{P}\left(\Gamma_{8}\right)$ (in $\left.\mathrm{eV}\right)$. The data are obtained for the theoretical equilibrium volume both with (SOC) and without (noSOC) spin-orbit coupling as well as for the experimental lattice parameter $\left(a^{\text {expt}}\right)$ with the account of SOC.

\begin{tabular}{lll}
\hline \hline & $\mathrm{K}_{3} \mathrm{Bi}$ & $\mathrm{Rb}_{3} \mathrm{Bi}$ \\
\hline noSOC & +0.057 & +0.036 \\
SOC & -0.35 & -0.40 \\
SOC at $a^{\text {expt }}$ & -0.34 & -0.16 \\
\hline \hline
\end{tabular}
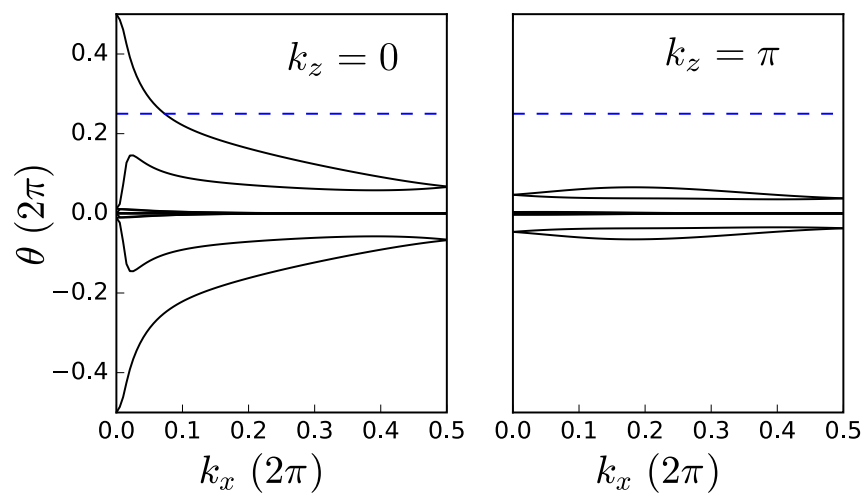

FIG. 5. The evolution of Wannier charge centers (WCCs) for $\mathrm{K}_{3} \mathrm{Bi}$ : (left) $k_{z}=0$, (right) $k_{z}=\pi$. A reference value is represented by the blue dashed line.

topological phase of $\mathrm{K}_{3} \mathrm{Bi}$ in spite of the semimetallic character of electronic structure. So the compound can be adiabatically transformed into an insulating phase with the same $Z_{2}$ indexes by a small uniaxial extension, which was shown for $\mathrm{KNa}_{2} \mathrm{Bi}$ [39].

\section{Changes induced by pressure}

To study the changes induced by hydrostatic compression, we calculated the electronic band dispersions of both compounds at different volumes. Figure 6 shows the evolution of electronic bands under pressure for $\mathrm{K}_{3} \mathrm{Bi}$. At low pressures the compound is still a semimetal with the inverted band order at the Brillouin zone center until the energy gap between $s$-type and $p$-like bands, $\varepsilon^{S}\left(\Gamma_{6}\right)-\varepsilon^{P}\left(\Gamma_{8}\right)$, vanishes. Figure 7 shows the variation of the energy gap with volume $\left(V / V_{0}\right)$ for both compounds. For comparison, the data for a ternary alkali bismuthide, $\mathrm{KNa}_{2} \mathrm{Bi}$, which crystallizes in the cubic $F m \overline{3} m$ structure [32,39] are also shown. Since the optimized and experimental lattice parameters for $\mathrm{Rb}_{3} \mathrm{Bi}$ differ noticeably, the energy gap in $\mathrm{Rb}_{3} \mathrm{Bi}$ as a function of $V / V_{\exp }$ is given in the inset.

Right at the point of topological transition $[P \approx 1.8 \mathrm{GPa}$ for both compounds, Fig. 6(b)], two of the bands form a conical dispersion crossed by the third band [Fig. 6(b)]. Upon further increase of pressure an energy gap appears between the valence and conduction bands and the topological semimetal turns into a conventional gapped semiconductor. Simultaneously, the band order around the $\Gamma$ point restores to normal when the $s$-type $\Gamma_{6}$ band is located above the $\Gamma_{8}$ bands composed of $p$-type Bi states [Fig. 6(c)]. A similar transition occurs in $\mathrm{KNa}_{2} \mathrm{Bi}$, a topological semimetal, which upon hydrostatic compression become a trivial semiconductors [39]. $\mathrm{Na}_{3} \mathrm{Bi}$ transforms to a regular insulator at $\sim 3.65 \mathrm{GPa}[10]$. Also, such semimetal-to-semiconductor topological transitions were reported in the crystals of $\mathrm{Hg}_{1-x} \mathrm{Cd}_{x}$ Te by changing cadmium concentration [13].

So, in the cubic phase of the $A_{3} \mathrm{Bi}(A=\mathrm{Na}, \mathrm{K}, \mathrm{Rb})$ compounds a 3D Dirac semimetal state is realized at the critical point of the topological phase transition between a topological semimetal and a normal band insulator by pressure-induced modifications of electronic structure (the so-called trivial Dirac semimetals [14]). Such a Dirac semimetal state features a 

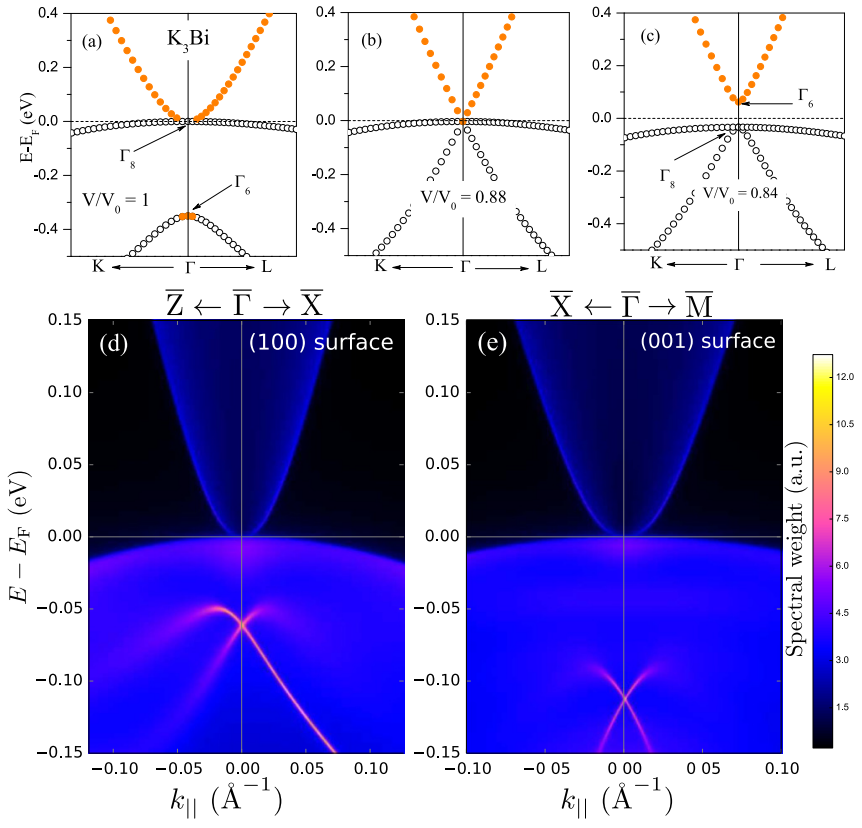

FIG. 6. (a)-(c) Evolution of the $\mathrm{K}_{3} \mathrm{Bi}$ electronic spectrum under hydrostatic lattice compression. Electronic bands near the $\Gamma$ point are shown for three different volumes: at $V_{0}(P=0$, a topological semimetal), at the point of topological transition $(P \approx 1.8 \mathrm{GPa})$, and at $P \approx 2.1 \mathrm{GPa}$ (a trivial semimetal). Open circles show the bands formed by $p$ states, while the bands composed mainly of $s$-type states are depicted by solid (orange) circles. (d),(e) Projected spectral functions for the $\mathrm{Bi}$ and $\mathrm{K}^{\mathrm{O}}$ terminated $(001)$ and $\mathrm{K}^{\mathrm{T}}$ terminated (100) surfaces of $\mathrm{K}_{3} \mathrm{Bi}$ at the equilibrium volume, $V_{0}$. The projection is shown by a color scale reflecting the localization in the outermost surface layer (in units of states/eV).

single 3D Dirac cone at the $\Gamma$ point, where the bulk band inversion occurs. Unlike the pressure-induced case, the Dirac states in the hexagonal $\mathrm{Na}_{3} \mathrm{Bi}$ and $\mathrm{K}_{3} \mathrm{Bi}$ show a pair of bulk

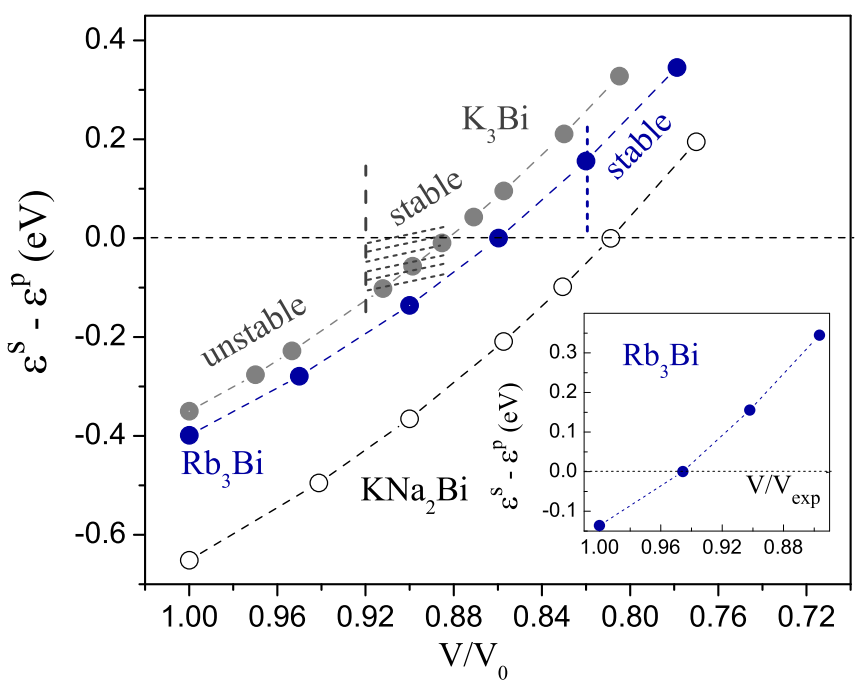

FIG. 7. Energy gap $\varepsilon^{S}\left(\Gamma_{6}\right)-\varepsilon^{P}\left(\Gamma_{8}\right)$ as a function of $V / V_{0}$. The positive gaps correspond to a topologically trivial phase when the band inversion disappears. The inset shows the energy gap in $\mathrm{Rb}_{3} \mathrm{Bi}$ as a function of $V / V_{\text {exp }}$.
Dirac nodes located on the rotation axis away from the $\Gamma$ point and protected by the rotational symmetry (the topological Dirac semimetal-metal) $[6,14,33]$. With respect to $\mathrm{Rb}_{3} \mathrm{Bi}$, it is a metal with an inverted band order in the bulk BZ center, but the Dirac band crossing on the rotational axis does not occur.

The topologically nontrivial character of semimetals is usually manifested in the existence of topological surface states. We calculated the surface electronic structure using $a b$ initio-based tight-binding (TB) formalism implemented in the WANNIER90 package $[40,41]$. The TB model Hamiltonian was constructed by projecting the bulk energy bands obtained in the first-principles calculation onto maximally localized Wannier functions (WFs). In our calculations, the spinor WF basis was chosen to be $\left|p_{x}^{\uparrow}\right\rangle,\left|p_{y}^{\uparrow}\right\rangle,\left|p_{z}^{\uparrow}\right\rangle,\left|p_{x}^{\downarrow}\right\rangle,\left|p_{y}^{\downarrow}\right\rangle,\left|p_{z}^{\downarrow}\right\rangle$ for $\mathrm{Bi}$ atoms and $\left|s^{\uparrow}\right\rangle,\left|s^{\downarrow}\right\rangle$ for $\mathrm{K}(\mathrm{Rb})$ atoms. The projected surface states were then obtained from the surface Green's function $\left(\hat{\mathcal{G}}_{\mu \nu}\right)$ [42-44]. $\hat{\mathcal{G}}_{\mu \nu}$ for the semi-infinite systems is defined as

$$
\left[(E+i \delta) \hat{\mathcal{I}}_{\mu \eta}-\hat{\mathcal{H}}_{\mu \eta}^{\text {surf }}\right] \hat{\mathcal{G}}_{\eta \nu}(E)=\hat{\mathcal{I}}_{\mu \nu}
$$

where $E$ is an energy parameter, $\delta$ is a small smooth parameter $\left(\delta=0.5 \times 10^{-3} \mathrm{eV}\right.$ in our calculation), and $\hat{\mathcal{I}}_{\mu \nu}$ is a unit matrix. $\mu, v$, and $\eta$ enumerate principal layers which interact as nearest neighbors. The surface spectral function, $A(E)$, was obtained from

$$
A(E)=-\frac{1}{\pi} \operatorname{Im} \operatorname{Tr} \hat{\mathcal{G}}_{00}(E) .
$$

The projected spectral functions calculated for the $\mathrm{Bi}$ and $\mathrm{K}^{\mathrm{O}}$ terminated $(001)$ and $\mathrm{K}^{\mathrm{T}}$ (100) surfaces of $\mathrm{K}_{3} \mathrm{Bi}$ (see Table I for notations) are shown in Figs. 6(d) and 6(e). The data are presented as a color intensity plot. The surface states with a Dirac-type crossing are clearly seen inside the projected valence bands on both surfaces presented.

In $\mathrm{K}_{3} \mathrm{Bi}$ the pressure-induced topological transition to a trivial state occurs when the cubic phase is already dynamically stable. Thus, at $T=0 \mathrm{~K}$ there is a pressure interval (see Fig. 7, the hatched area) where the compound is still a topological semimetal and is already dynamically stable $\left(0.92 \geqslant V / V_{0} \geqslant 0.88,0.95 \mathrm{GPa} \leqslant P \leqslant 1.8 \mathrm{GPa}\right)$. Unlike $\mathrm{K}_{3} \mathrm{Bi}$, the cubic phase of $\mathrm{Rb}_{3} \mathrm{Bi}$ stabilizes at $T=0 \mathrm{~K}$ only after the transition to a trivial semiconductor has occurred (at $\left.V / V_{0} \approx 0.82, P \approx 2.8 \mathrm{GPa}\right)$.

\section{CONCLUSION}

We have performed an ab initio study of the effect of pressure on electronic and vibrational properties of alkali bismuthides, $\mathrm{K}_{3} \mathrm{Bi}$ and $\mathrm{Rb}_{3} \mathrm{Bi}$, in the cubic $F m \overline{3} m$ structure. We find that at zero pressure the cubic compounds are topologically nontrivial with the inverted band order at the $\Gamma$ point and undergo a transition to a conventional semiconductor (metal) state upon hydrostatic compression. Simultaneously, the sequence of bands restores to normal.

It is also shown that the cubic structure of $\mathrm{K}_{3} \mathrm{Bi}$ and $\mathrm{Rb}_{3} \mathrm{Bi}$ at low temperatures (i) is dynamically unstable and (ii) can be 
stabilized by pressure. In $\mathrm{K}_{3} \mathrm{Bi}$, the stabilization occurs before the transition to a trivial semiconductor so that there is a pressure interval where the cubic phase is stable and the compound is still topologically nontrivial. Unlike $\mathrm{K}_{3} \mathrm{Bi}$, the cubic phase of $\mathrm{Rb}_{3} \mathrm{~B}$ stabilizes after the transition to a trivial semiconductor has occurred.

\section{ACKNOWLEDGMENTS}

This work has been supported by the Spanish Ministry of Science and Innovation (Grant No. FIS2016-75862-P), Tomsk State University Academic D.I. Mendeleev Fund Program (Research Grant No. 8.1.01.2017), and Saint Petersburg State University (Project No. 15.61.202.2015).
[1] Z. Wang, Y. Sun, X.-Q. Chen, C. Franchini, G. Xu, H. Weng, X. Dai, and Z. Fang, Phys. Rev. B 85, 195320 (2012).

[2] Z. K. Liu, B. Zhou, Y. Zhang, Z. Wang, H. Weng, D. Prabhakaran, S.-K. Mo, Z. X. Shen, Z. Fang, X. Dai, Z. Hussain, and Y. L. Chen, Science 343, 864 (2014).

[3] S.-Y. Xu et al., Phys. Rev. B 92, 075115 (2015).

[4] S. M. Young, S. Zaheer, J. C. Y. Teo, C. L. Kane, E. J. Mele, and A. M. Rappe, Phys. Rev. Lett. 108, 140405 (2012).

[5] E. V. Gorbar, V. A. Miransky, I. A. Shovkovy, and P. O. Sukhachov, Phys. Rev. B 91, 235138 (2015).

[6] A. Narayan, D. Di Sante, S. Picozzi, and S. Sanvito, Phys. Rev. Lett. 113, 256403 (2014).

[7] D. E. Sands, D. H. Wood, and W. J. Ramsey, Acta Cryst. 16, 316 (1963).

[8] K. A. Chuntonov, S. P. Yatsenko, A. N. Kuznetsov, S. I. Alyamovskii, and K. K. Abrashev, Kristallografiya 22, 641 (1977).

[9] M. E. Leonova, L. G. Sevastyanova, O. K. Gulish, and K. P. Burdina, Inorg. Mater. 37, 1270 (2001).

[10] X. Y. Cheng, R. H. Li, D. Z. Li, Y. Y. Li, and X.-Q. Chen, Phys. Chem. Chem. Phys. 17, 6933 (2015).

[11] X. Cheng, R. Li, D. Li, Y. Li, and X.-Q. Chen, Phys. Rev. B 92, 155109 (2015).

[12] W. Feng, W. Zhu, H. H. Weitering, G. M. Stocks, Y. Yao, and D. Xiao, Phys. Rev. B 85, 195114 (2012).

[13] M. Orlita, D. M. Basko, M. S. Zholudev, F. Teppe, W. Knap, V. I. Gavrilenko, N. N. Mikhailov, S. A. Dvoretskii, P. Neugebauer, C. Faugeras, A.-L. Barra, G. Martinez, and M. Potemski, Nat. Phys. 10, 233 (2014).

[14] B.-J. Yang and N. Nagaosa, Nat. Commun. 5, 4898 (2014).

[15] S. G. Louie, K.-M. Ho, and M. L. Cohen, Phys. Rev. B 19, 1774 (1979).

[16] B. Meyer, C. Elsässer, F. Lechermann, and M. Fähnle, FORTRAN90, Program for Mixed-Basis-Pseudopotential Calculations for Crystals (Max-Planck-Institut für Metallforschung, Stuttgart).

[17] R. Heid and K.-P. Bohnen, Phys. Rev. B 60, R3709 (1999).

[18] J. P. Perdew, K. Burke, and M. Ernzerhof, Phys. Rev. Lett. 77, 3865 (1996).

[19] D. Vanderbilt, Phys. Rev. B 32, 8412 (1985).

[20] S. Baroni, S. de Gironcoli, A. Dal Corso, and P. Giannozzi, Rev. Mod. Phys. 73, 515 (2001).

[21] L. Kleinman, Phys. Rev. B 21, 2630 (1980).
[22] R. Heid, K.-P. Bohnen, I. Yu. Sklyadneva, and E. V. Chulkov, Phys. Rev. B 81, 174527 (2010).

[23] P. Giannozzi, S. de Gironcoli, P. Pavone, and S. Baroni, Phys. Rev. B 43, 7231 (1991).

[24] A. A. Soluyanov and D. Vanderbilt, Phys. Rev. B 83, 035108 (2011).

[25] R. Yu, X. L. Qi, A. Bernevig, Z. Fang, and X. Dai, Phys. Rev. B 84, 075119 (2011).

[26] A. Alexandradinata, X. Dai, and B. A. Bernevig, Phys. Rev. B 89, 155114 (2014).

[27] G. Gnutzman, F. W. Dorn, and W. Klemm, Z. Anorg. Allg. Chem 309, 210 (1961).

[28] G. Kresse and J. Furthmüller, Phys. Rev. B 54, 11169 (1996).

[29] D. Liu and J. Nocedal, Math. Program. B 45, 503 (1989).

[30] G. Brauer and E. Zintl, Z. Phys. Chem. 37B, 323 (1937).

[31] M. Mansmann, Z. Kristallogr. 122, 399 (1965).

[32] H. Kerber, H.-J. Deiseroth, and R. Walther, Z. Kristallogr.-NCS 213, 501 (1998).

[33] X. Cheng, R. Li, Y. Sun, X.-Q. Chen, D. Li, and Y. Li, Phys. Rev. B 89, 245201 (2014).

[34] G. S. Jenkins, C. Lane, B. Barbiellini, A. B. Sushkov, R. L. Carey, F. Liu, J. W. Krizan, S. K. Kushwaha, Q. Gibson, T.-R. Chang, H.-T. Jeng, H. Lin, R. J. Cava, A. Bansil, and H. D. Drew, Phys. Rev. B 94, 085121 (2016).

[35] M. Tegze and J. Hafner, J. Phys.: Condens. Matter 4, 2449 (1992).

[36] A. D. Becke, Phys. Rev. A 38, 3098 (1988).

[37] J. Heyd, G. E. Scuseria, and M. Ernzerhof, J. Chem. Phys. 118, 8207 (2003).

[38] P. J. Hasnip, K. Refson, M. I. J. Probert, J. R. Yates, S. J. Clark, and C. J. Pickard, Phil. Trans. R. Soc. A 372, 20130270 (2014).

[39] I. Yu. Sklyadneva, I. P. Rusinov, R. Heid, K.-P. Bohnen, P. M. Echenique, and E. V. Chulkov, Sci. Rep. 6, 24137 (2016).

[40] N. Marzari and D. Vanderbilt, Phys. Rev. B 56, 12847 (1997).

[41] W. Zhang, R. Yu, H.-J. Zhang, X. Dai, and Z. Fang, New J. Phys. B 12, 065013 (2010).

[42] M. P. Lopez Sancho, J. M. Lopez Sancho, and J. Rubio, J. Phys. F 14, 1205 (1984).

[43] M. P. Lopez Sancho, J. M. Lopez Sancho, and J. Rubio, J. Phys. F 15, 851 (1985).

[44] J. Henk and W. Schattke, Comput. Phys. Commun. 77, 69 (1993). 